\title{
The Dieulafoy gastric malformation: an under-recognized cause of massive upper gastrointestinal haemorrhage
}

\author{
M. Welch and E.M. Hoare \\ Department of Surgery, Trafford General Hospital, Moorside Road, Davyhulme, Manchester, M31 3SL, \\ $U K$
}

\begin{abstract}
Summary: The Dieulafoy gastric malformation is a rare cause of upper gastrointestinal haemorrhage. When no obvious bleeding lesion is seen at laparotomy this diagnosis ought to be considered. Three such cases were identified and treated with simple underrunning of the lesion with no mortality and minimal morbidity. Follow-up endoscopy in each patient showed complete healing of the lesion.
\end{abstract}

\section{Introduction}

We present three cases of massive upper gastrointestinal haemorrhage from the Dieulafoy vascular malformation. ${ }^{1}$ Fewer than 150 such cases have been reported in the literature, yet we have seen three cases in a 4-month period in a district general hospital. We feel that this is an underrecognized cause of massive upper gastrointestinal haemorrhage, and one which must be carefully sought in the absence of an obvious bleeding lesion.

\section{Case reports}

\section{Case 1}

A 59 year old man was admitted with an 8-hour history of periumbilical pain, haematemesis and malaena. He had a history of dyspepsia over the preceding 5 months, and his alcohol intake was 8 pints of beer each day. On examination he was pale and sweaty with a tachycardia of 130 beats/minute and blood pressure $115 / 65 \mathrm{mmHg}$. Haemoglobin estimation was $13.3 \mathrm{mmol} / 1$, urea $14.6 \mathrm{mmol} / 1$. He was resuscitated with intravenous fluids, catheterized, and transfused 3 units of blood.

Four hours after admission he had a further massive haematemesis, passed fresh malaena, and became shocked. Urgent gastroscopy was performed: there was no evidence of oesophogeal varices, but the stomach was full of fresh blood and

Correspondence: M. Welch, F.R.C.S. (Ed), S3 Records, Manchester Royal Infirmary, Oxford Road, Manchester, M13 9WL, UK

Accepted: 25 January 1991 clot, preventing visualization of any lesion there. Laparotomy was performed through an upper mid-line incision. An initial duodenotomy revealed no evidence of duodenal ulceration, and therefore a long anterior gastrotomy was performed. Despite a careful search no bleeding lesion was identified, but blood was seen to be coming from the region of the cardia. By palpation a small abnormality $1 \mathrm{~cm}$ distal to the cardia on the lesser curve was identified, and with careful positioning of retractors a split in the mucosa with a bleeding vessel projecting through was seen. The lesion was treated by underrunning with several linen sutures.

The patient made an excellent post-operative recovery, having received a 10 -unit blood transfusion in all, and was discharged after 8 days. A follow-up gastroscopy after 2 months showed no evidence of the lesion.

\section{Case 2}

A 49 year old man presented with a 12-hour history of haematemesis, malaena and dizziness. On examination he was pale but not shocked. Haemoglobin estimation was $13.6 \mathrm{mmol} / \mathrm{l}$, with a urea of $16.4 \mathrm{mmol} / \mathrm{l}$. He was resuscitated with intravenous fluids and 2 units of blood. Urgent gastroscopy was performed, excluding oesophageal varices and duodenal ulceration, but fresh blood and clot prevented adequate views of the gastric mucosa. He had further episodes of malaena, and after 4 more units of blood, haemoglobin estimation was only $8.0 \mathrm{~g} / \mathrm{dl}$. Laparotomy was therefore performed, via an upper midline incision. Opening the stomach by an anterior gastrotomy all blood and clot was evacuated, and no obvious bleeding lesion was seen. The region of the cardia was therefore 
palpated, and an abnormality felt $2 \mathrm{~cm}$ distal to the cardia on the lesser curve side. This was seen to be a split in the mucosa with a large artery in its base, a branch of which was projecting through the mucosal defect and bleeding continuously. The lesion was underrun with several linen sutures. Twelve units of blood had been transfused in all.

The patient made an excellent recovery and was discharged on the eighth post-operative day. At follow up gastroscopy 2 months later, the area was a little erythematous and one linen stitch was still present, but the lesion had otherwise healed.

\section{Case 3}

A 78 year old woman presented with a 2-day history of indigestion and sudden collapse. On examination she was pale and shocked. Haemoglobin estimation was $5.6 \mathrm{mmol} / 1$ with a urea of $24.0 \mathrm{mmol} / \mathrm{l}$. She was quickly resuscitated with intravenous fluids and 4 units of blood. Twelve hours later she had a massive haematemesis associated with further hypotension, and a laparotomy was therefore performed. The duodenum was scarred but there was no bleeding lesion. A long anterior gastrotomy was made and the stomach emptied of fresh blood and clot, but no obvious lesion was seen. An abnormality near the cardia was identified by palpation, and when visualized was a split in the mucosa $1 \mathrm{~cm}$ distal to the cardia on the lesser curve, with a large blood vessel in its base capped by some thrombus. The lesion was underrun with several linen sutures.

She made an uneventful post-operative recovery, having received 9 units of blood in all, and was discharged after 17 days. Follow-up gastroscopy 2 months later showed complete healing of the lesion.

\section{Discussion}

Although originally described by Gallard in 1884, the Dieulafoy gastric erosion or vascular malformation was so named in 1896 . The lesion consists of a small mucosal erosion with a cap of thrombus overlying a ruptured submucosal vessel, with a predilection for the lesser curve $1-3 \mathrm{~cm}$ from the cardia. The lesion was originally thought to be caused by an aneurysm of a submucosal vessel, but more recent reports believe that the affected vessel represents part of a localized congenital malformation, with possibly focal pressure from the enlarged vessel on the overlying gastric mucosa, precipitating erosion of the mucosa and exposed vascular wall. $^{2}$ However, histological examination of such lesions has not shown the typical changes of endarteritis which are usually seen in response to peptic ulceration. ${ }^{3}$ Voth $^{4}$ believes that the primary lesion is the superficial erosion which occurs in a part of the stomach where an abnormally large artery happens to run through the submucosa. This may explain why the majority of the lesions arise along the proximal part of the lesser curve of the stomach, where the arrangement of blood vessels in the submucosa differs from the rest of the stomach. $^{5}$

The patient typically presents with massive haematemesis and melaena, with circulatory shock. The reported age range is 20 months to 93 years, with the majority between the ages of 50-70 years. ${ }^{6}$ The male:female ratio is $2: 1$. There may be a previous history of peptic ulceration or alcohol abuse, but generally there are no obvious pointers to the diagnosis from the history and examination.

Urgent gastroscopy is required and may provide the diagnosis but often one is faced with performing a laparotomy without knowing the source of the bleeding. A history of non-steroidal antiinflammatory drug ingestion or previous duodenal ulceration would prompt one to perform an initial duodenotomy, but if no lesion is found an anterior gastrotomy should be made, and the gastric mucosa carefully examined for the source of bleeding. This usually requires the help of at least one assistant holding two retractors inside the stomach in order that the region of the fundus and cardia may be visualized. If no lesion is seen, a finger is inserted into the distal oesophagus and gently brought back into the stomach, carefully feeling the mucosa. In each of the three cases described, a slight mucosal abnormality was felt on the lesser curve just distal to the cardio-oesophageal junction, and with repositioning of the retractors and the use of suction the mucosal defect and bleeding lesion was visualized.

The literature recommends wedge excision of the Dieulafoy vascular malformation; ${ }^{5}$ however, the reported morbidity and mortality is relatively high.

Our experience suggests that simple underrunning of the bleeding lesion with 3 or 4 linen sutures is the correct emergency treatment of this bleeding lesion. Each of our patients made an uneventful post-operative recovery and endoscopic follow-up in all of them has shown the lesions to be healed.

Patients may afterwards be treated with $\mathrm{H} 2$ antagonists; the small number of patients who require subsequent elective surgery will be determined by clinical and endoscopic criteria.

In conclusion, the Dieulafoy gastric malformation is a cause of massive upper gastrointestinal haemorrhage which, in the absence of any obvious bleeding lesion, must be carefully sought. Digital palpation in the region of the cardia will identify the lesion which is not immediately visible. Simple suture ligation of the lesion is the treatment of choice, with mandatory endoscopic follow-up. 


\section{References}

1. Dieulafoy, G. Exulceratio simplex. L'intervention chirurgicale dans les hematemeses foundroyantes consecutives a l'exulceration simple de l'estomac. Bull Acad Med 1898, 49: 49-84.

2. Goldman, R.L. Submucosal arterial malformation ('aneurysm') of the stomach with fatal haemorrhage. Gastroenterology 1964, 46: 689-694.

3. Juler, G.L., Labitzke, H.G., Lamb, R. \& Allen, R. The pathogenesis of Dieulafoy's gastric erosion. Am J Gastroenterol 1984, 79: 195-200.

4. Voth, D. Zur Pathogenese ungewohnlicher, Arterieller Magenblutungen. Med Welt 1962; 19: 1095- 1097.

5. Barlow, T.E., Bently, F.H.\& Walder, D.N. Arteries, veins and arteriovenous anastomoses in the human stomach. Surg Gynecol Obstet 1951, 93: 657-671.

6. Veldhyzen Van Zanten, S.J.O., Bartelsman, J.F.W.M., Schipper, M.E.I. \& Tytgat, G.N.J. Recurrent massive haematemesis from Dieulafoy vascular malformations - a review of 101 cases. Gut 1986, 27: 213-222.

.

